\title{
Neural Predictors of Within-Subject Fluctuations in Attentional Control
}

\author{
Andrew B. Leber \\ Department of Psychology, University of New Hampshire, Durham, New Hampshire 03824
}

Whether salient objects automatically capture attention has long been the subject of considerable controversy. A possible resolution, investigated in this functional magnetic resonance imaging (fMRI) study, is that observers vacillate between periods when attention capture is robust and when it is minimal. Human observers searched static displays for a target circle among nontarget squares; an irrelevant color singleton distractor appeared on $50 \%$ of trials. Behavioral results showed a distraction effect in which response times to distractor-present trials were slower than for distractor-absent trials. fMRI results confirmed that this distraction effect not only fluctuated within experimental sessions, but the momentary degree of distraction could be predicted in advance by pretrial activity in middle frontal gyrus. A second experiment ruled out an alternative account by which participants achieved resistance to capture by trading off search efficiency. Together, these data reveal that observers frequently exert the capacity to resist attentional distraction, although they do not to sustain this capacity for long periods of time.

\section{Introduction}

The environment is filled with an incalculable abundance of visual information, yet our capacity to process this information is severely limited. As a result, the vast majority of input we receive fails to reach awareness, and we are thus faced with the challenge of efficiently attending behaviorally important information while avoiding wasteful processing of irrelevant information. To meet this challenge, human information processing uses two components of attentional control: stimulus-driven processing, which acts to prioritize information on the basis of low-level properties, and goal-driven processing, which acts to prioritize information based on its degree of relevance to the current task. Although there is broad agreement on the existence of these components of control, the manner in which they interact to determine overall attentional priority has been the source of considerable controversy. Some have argued that the stimulus-driven component exerts dominance in determining priority (Yantis and Jonides, 1984; Theeuwes, 1992; Franconeri and Simons, 2003), whereas others have assigned dominance to the goal-driven component (Folk et al., 1992). Accounts of goal-driven dominance have been supported by demonstrations that interference by salient, irrelevant distractors can be reduced or eliminated (Bacon and Egeth, 1994; Serences et al., 2005; Leblanc et al., 2008; Lien et al., 2008), yet a parallel stream of studies continues to consistently show sizable interference effects (Theeuwes, 1991) (see also Theeuwes, 1992, 1994, 2004; Hickey et al., 2006; Belopolsky et al., 2007).

Received Jan. 19, 2010; revised June 7, 2010; accepted July 8, 2010.

This research was funded by the University of New Hampshire. I thank T. Hickey, K. Martin, and C. McMurray for scanning assistance, D. Lartaud and J. Lechak for additional assistance with data collection, and M. Chun, N. Turk-Browne, and J. Golomb for helpful comments and suggestions.

Correspondence should be addressed to Andrew B. Leber, Department of Psychology, University of New Hampshire, Conant Hall, 10 Library Way, Durham, NH 03824. E-mail: andrew.leber@unh.edu.

DOI:10.1523/JNEUROSCI.0809-10.2010

Copyright $\odot 2010$ the authors $\quad 0270-6474 / 10 / 3011458-08 \$ 15.00 / 0$
In this study, a reconciliation of the opposing bodies of evidence is proposed, in which performance in a typical experimental session is sometimes dominated by stimulus-driven processing and sometimes by goal-driven processing. That is, the ability to resist distraction could undergo fluctuations from moment to moment. Such fluctuations, should they exist, would have been overlooked in previous research because conventional analyses average trials across sessions; that is, session data including moments of both negligible and robust distraction, when averaged, should yield a net positive distraction effect. This study peered within experimental sessions via a functional magnetic resonance imaging (fMRI) analysis technique in which temporal variations in neural activity are used to illuminate intraindividual variations in behavior (Pessoa et al., 2002; Sapir et al., 2005; Turk-Browne et al., 2006; Weissman et al., 2006; Hahn et al., 2007; Eichele et al., 2008; Leber et al., 2008). In particular, a data-driven, whole-brain regression approach was used, in which fMRI activity preceding each trial was used to predict behavioral performance (Leber et al., 2008). The objectives were to (1) confirm that susceptibility to attention capture does fluctuate from moment to moment, (2) predict these fluctuations in advance via pretrial fMRI activity, and (3) determine which brain regions mediate such fluctuations.

\section{Materials and Methods}

\section{Experiment 1: whole-brain analysis}

\section{Participants}

Fifteen neurologically healthy individuals (mean age, 25.1 years; seven females), with normal or corrected-to-normal visual acuity and normal color vision, participated in exchange for monetary compensation. Participation included a brief ( $<10 \mathrm{~min}$ ) behavioral practice session, followed by $\sim 60 \mathrm{~min}$ of anatomical and functional scans. Procedures were approved by the Human Investigation Committee of the Yale School of Medicine and the Institutional Review Board of the University of New Hampshire. 

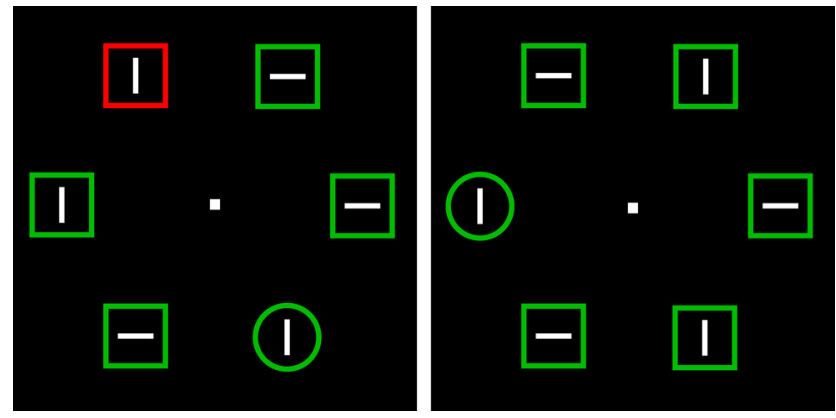

Figure 1. Representation of trial stimuli. Participants searched for the circle and reported the orientation of the line segment inside of it. Half of the trials contained an irrelevant color singleton distractor that could never be the target (left), although the remaining trials did not (right). For additional details, see Materials and Methods.

\section{Stimulus presentation and behavioral measurement}

Stimuli were generated via an Apple MacBook computer, using MATLAB software (MathWorks) with Psychophysics Toolbox extensions (Brainard, 1997; Pelli, 1997). During fMRI scanning, stimuli were displayed via a liquid crystal display projector onto a screen mounted in the rear of the scanner bore, which participants viewed via an angled mirror attached to the head coil.

Participants performed a feature-based search for a circle among squares, as shown in Figure 1, and they were instructed to indicate whether a vertical or horizontal line segment appeared inside the circle. Vertical lines were reported via a button press by the right index finger, and horizontal lines were reported via a button press by the right middle finger. A fiber-optic button box was used to collect responses. Squares subtended $2.34^{\circ}$ visual angle in width and circles subtended $2.69^{\circ}$ in diameter; each shape was centered at $5.85^{\circ}$ eccentricity from fixation and drawn with a stroke of $0.20^{\circ}$. Each display consisted of six total shapes, all distributed evenly on the circumference of an imaginary circle. Displays were symmetrical about the vertical and horizontal axes, with three shapes appearing in the right hemifield and three in the left. The orientation of the line segment occupying each shape was selected randomly for each object on each trial with the constraint that half of the search items contained a vertical line and the other half a horizontal line. These lines were centered inside the shapes, each subtending $1.17^{\circ}$, with a stroke of $0.20^{\circ}$, and always colored white.

The display objects (target and nontarget shapes) were drawn in two colors, red and green. Half of the participants were assigned red as the "standard" color and green as the distracting "singleton" color, and this was reversed for the remaining participants. Half of the trials were "distractor absent," in which all items were presented in the standard color; the other half of the trials were "distractor present," in which all but one of the items were presented in the standard color, and the remaining item was presented in the singleton color. Two additional variables, target location and distractor-to-target separation, were manipulated for control purposes: target location ensured that targets appeared with equal frequency at the six search locations, and distractor-target separation ensured that for each target location, distractors appeared with equal frequency at the remaining five display locations.

Trials were time-locked to the acquisition of whole-brain volumes, which were taken in $1500 \mathrm{~ms}$ increments. The minimum trial-to-trial onset was set at $3000 \mathrm{~ms}$, and, in conforming to a rapid event-related design (Dale and Buckner, 1997), trial spacing was temporally jittered such that half of the trials had an additional $1500 \mathrm{~ms}$ blank interval inserted before the trial onset, whereas the remaining trials did not. Thus, each trial onset followed the previous trial onset by 3000 or $4500 \mathrm{~ms}$. Distractor presence (two levels), target location (six levels), distractortarget distance (five levels), and trial spacing (two levels) were factorially crossed, yielding 120 trials per run, which were presented in random order.

The first $10.5 \mathrm{~s}$ of each run consisted of only a white fixation circle $\left(0.29^{\circ}\right.$ diameter $)$, a period that allowed for fMRI signal stabilization. Trial events began after this period. At $1000 \mathrm{~ms}$ before each trial onset, the fixation briefly enlarged by $67 \%$ for $200 \mathrm{~ms}$ to indicate the impending trial stimulus was about to appear. At each trial onset, search arrays were added to the display for $200 \mathrm{~ms}$, at which point they were removed, leaving only fixation. Responses were collected for up to $1500 \mathrm{~ms}$ after the search array onset. At all other times, only fixation was present, and participants were instructed to remain fixated across the duration of each run. Feedback was provided by the experimenter after each run. Participants completed four scanning runs, each $\sim 8 \mathrm{~min}$ in duration.

\section{fMRI acquisition}

MRI scanning was performed with a 3.0 Tesla Siemens Trio scanner, located at the Magnetic Resonance Research Center at the Yale School of Medicine. An eight-channel receiver array head coil was used. For each participant, after an initial anatomical localizer, a high-resolution T1-weighted anatomical image was acquired with a three-dimensional magnetization-prepared rapid-acquisition gradient echo sequence. Then, 34 axial slices were defined ( $3.5 \mathrm{~mm}$ thickness, no gap), parallel to the anterior commissure-posterior commissure line, covering the whole brain, and a second anatomical image was acquired, using a T1 fast, low-angle shot sequence. Using the same 34 -slice orientation, $\mathrm{T} 2{ }^{*}$ weighted functional runs were then performed [repetition time (TR), $1500 \mathrm{~ms}$; echo time, 25 ; flip angle, $90^{\circ}$; $64 \times 64$ matrix with $3.5 \times 3.5 \mathrm{~mm}$ in-plane resolution].

\section{fMRI data preprocessing}

BrainVoyagerQX software was used for preprocessing (Brain Innovation). For each subject, the first seven volumes of each functional run were discarded (accounting for $10.5 \mathrm{~s}$ of signal stabilization), and the data were subsequently motion corrected and slice-time corrected. Data were then spatially smoothed with a $4 \mathrm{~mm}$ full-width half-maximum isotropic Gaussian kernel and were then subjected to linear trend removal. Because of the possibility that fluctuations in attentional control could occur at slow temporal frequencies, the data were not temporally high-pass filtered. Finally, the data were transformed to Talairach space (Talairach and Tournoux, 1988) and resampled at a voxel size of $3 \times 3 \times 3 \mathrm{~mm}$.

\section{fMRI whole-brain analysis: predicting behavior from pretrial signal}

In structuring an analysis to predict fluctuations in behavior, it is important to draw a distinction between true variations in attentional control versus variations in performance resulting from incidental aspects of the trial stimuli. To illustrate by example, consider that distractor interference on a given trial $(n)$ is known to be smaller when the distractor on the previous trial $(n-1)$ appeared in the same location, putatively because of persisting inhibition of the distractor location (Kumada and Humphreys, 2001). Here, the measured reduction in response time (RT) interference effects is not the result of the observer bringing greater goaldriven control into the trial; rather, it results from a fortuitous, idiosyncratic aspect of the stimulus display (one that happens with somewhat regular frequency, i.e., 1/display set size, if the distractor appears at each location with equal frequency). Problems associated with incidental aspects of the trial stimuli are particularly insidious, because all manner of intertrial effects have been shown to modulate attention capture (Olivers and Humphreys, 2003; Lamy et al., 2005, 2008; Pinto et al., 2005; Müller et al., 2009). Because the aim was to predict fluctuations in attentional control rather than variations in performance attributable to benefits/ costs brought on by incidental aspects of stimuli, the current study predicted RT from the pretrial signal (Leber et al., 2008). Pretrial signal is preferred because it is sampled before neural activity can possibly be responding to (and thus representing components of) any incidental aspect of the upcoming stimulus presentation. A three-step description of how pretrial signal was defined, calculated, and used to predict behavior is summarized below.

Defining pretrial signal. Pretrial signal was defined as the blood oxygenation level-dependent (BOLD) activity collected from the single volume acquisition before each trial onset. Note that, because of the sluggish nature of the hemodynamic response, pretrial signal is likely to mostly reflect neural activity from several seconds before the trial onset rather than immediately before it. Thus, a potential drawback to this approach is poor sensitivity to fluctuations at higher temporal frequencies, one that 
is mitigated by the previously discussed benefits of sampling only neural activity unrelated to upcoming trial events.

Preparing $f M R I$ data for pretrial signal analysis. The goal was to discover brain regions whose baseline fluctuations in BOLD activity could predict attentional control (i.e., moment-to-moment susceptibility to distraction). One potential roadblock to this pursuit, however, is that brain regions undergoing control-related fluctuations also likely contain other components of BOLD variability that could contribute significant noise to the measurement. Consideration of trial spacing illustrates this point; because of known properties of the hemodynamic response, the BOLD signal $6 \mathrm{~s}$ after a trial onset (i.e., when the evoked response is at peak) is typically greater than the BOLD signal $10 \mathrm{~s}$ after a trial onset (i.e., when the signal is returning to baseline). As a result, when trials are spaced closely together, as in a rapid event-related design, pretrial signal will necessarily be determined in part by the onset time of the previous trial. Critically, such variance attributable to trial spacing, if not accounted for, could undermine attempts to assess fluctuations in attentional control. Another variable, previous trial type is also likely to influence the pretrial signal. This is because it is expected that some brain regions exhibit a greater peak response to distractor-present than to distractor-absent trials, and the peak of the previous trial is expected to affect the pretrial signal.

To overcome the potential deleterious effects of recent trial history on the proposed analysis (i.e., trial spacing and previous trial type), variance associated with these factors was removed. First, a linear model was fitted to the BOLD time course of each run, based on the mean evoked BOLD response to each trial type. Then, using multiple regression, the variance explained by this model was partialed out from the BOLD time course, and the pretrial analyses were subsequently performed on the residual BOLD time course (Weissman et al., 2006; Leber et al., 2008). Consequently, the pretrial signal values extracted from the residual time course data should have reflected variations in the signal that were not systematically biased by the temporal separation between trials or previous trial types.

The regression model was implemented to independently fit each time point of the trial-evoked hemodynamic response, thus making no assumptions about the shape of the response function (Dale, 1999). Twelve "candlestick" predictors were established for each of the main experimental conditions (distractor-present and distractor-absent trials) as well as for error trials, with each candlestick corresponding to one time point after trial onset; given 12 consecutive candlesticks and a $1.5 \mathrm{~s} \mathrm{TR}$, an $18 \mathrm{~s}$ duration, posttrial onset was thus modeled. Beyond the 36 predictors described above, the regression model also included eight predictors for additional sources of variance from the BOLD time courses in each run: (1) six parameters obtained from the motion correction procedure, (2) BOLD signal measured from a region in deep white matter, and (3) mean "global" BOLD signal, averaged across all voxels in the whole-brain dataset. These steps were taken to remove spurious sources of variance attributable to scanner and motion artifacts, respiration, and other factors deemed unrelated to neural activity and thus increase the probability of observing cognitively meaningful fluctuations in the BOLD signal (Fox et al., 2005; Vincent et al., 2006).

The regression partialed out all the above predictors from the BOLD time course in each voxel, for each run, for each participant, leaving "residual BOLD time courses," which were then $z$-transformed and used for additional analysis.

Predicting RT from pretrial signal. To address the main goal of predicting behavior from neural activity, two new linear regression analyses were performed, separately for each participant and within each voxel. After selecting and pooling pretrial signal values (from the residual BOLD time courses) across all runs, pretrial signal was defined as a predictor for RT, separately for distractor-present and distractor-absent trials. Each regression yielded a $\beta$, or slope, coefficient, which represents the degree to which RT changed as a function of pretrial signal. The slope coefficients for distractor-present and distractor-absent conditions from each participant were then entered into within-subject $t$ tests, separately for each voxel. Thus, a single whole-brain statistical parametric map of $t$ scores represented the degree to which the activity of each voxel predicted the distractor interference effect. This map was then transformed, via interpolation, to $1 \mathrm{~mm}^{3}$ isotropic voxel resolution. To determine significant clusters of voxels, a single-voxel threshold was set at $t_{(14)}=2.977$, $p<0.01$; then, by applying a spatial cluster threshold of $270 \mathrm{~mm}^{3}$, the false probability rate of identifying significant clusters was $p=0.029$. This estimate was reached by performing 2000 Monte Carlo simulations of whole-brain statistical maps, using the cluster threshold estimator of BrainVoyager (Goebel et al., 2006).

\section{Experiment 2: independent validation and display set size manipulation}

Methods were the same as in experiment 1, unless noted otherwise.

\section{Participants}

Sixteen new individuals (eight females) with a mean age of 26.3 years participated in this experiment.

\section{Stimulus presentation and behavioral measurement}

Search displays contained either 6 or 10 objects. To minimize visual crowding in the larger display set size, objects were slightly reduced in size compared with experiment 1 ; squares now subtended $1.95^{\circ}$ in width, circles subtended $2.24^{\circ}$ in diameter, and vertical/horizontal line segments appearing inside the shapes subtended $0.98^{\circ}$, although as in experiment 1 , all retained a stroke of $0.20^{\circ}$ and each shape was centered at $5.85^{\circ}$ eccentricity from fixation. At both display set sizes, objects were arranged symmetrically about the vertical and horizontal axes, with half of the items appearing in the left hemifield and half in the right hemifield. Minimum trial-to-trial onset was now set at $4000 \mathrm{~ms}$, to accommodate a longer TR (see below, fMRI acquisition and preprocessing), and trial spacing was temporally jittered such that half of the trials were preceded by a $2000 \mathrm{~ms}$ blank period, whereas the remaining trials were not. Thus, each trial onset followed the previous trial onset by 4000 or $6000 \mathrm{~ms}$.

Display set size (two levels), distractor presence (two levels), and trial spacing (two levels) were factorially crossed to create eight minimum conditions. Each of these conditions was repeated 12 times to yield 96 trials per run, which were presented in randomized order. Target location and distractor-to-target distance could not be included in the factorial design because of run-length constraints, and they were now determined randomly on each trial. Each run began with a $10 \mathrm{~s}$ fixation presentation to allow for fMRI signal stabilization, after which the trial presentations began. Participants completed five scanning runs, each $\sim 8$ min in duration, with the exception of one participant, who only completed four runs as a result of technical problems.

\section{fMRI acquisition and preprocessing}

As in experiment 1, the first 12 participants were scanned at the Magnetic Resonance Research Center at the Yale School of Medicine. The remaining participants were scanned on a 3 Tesla Siemens Tim Trio at the Center for Brain Science at Harvard University. Imaging parameters at Yale School of Medicine were the same as in experiment 1, except the functional runs now used a $2000 \mathrm{~ms}$ TR. At Harvard University, a 32channel head coil was used, and functional runs used the same imaging parameters used at Yale School of Medicine, except 31 slices were acquired. For all participants, the first five volumes of each functional run were discarded during preprocessing, accounting for the $10 \mathrm{~s}$ of signal stabilization.

\section{fMRI region of interest analysis}

This experiment focused, via a region of interest (ROI) analysis, on left middle frontal gyrus (MFG), as defined by the independently collected data in experiment 1 (see Results). A three-dimensional spherical boundary was created, encompassing a volume of $123 \mathrm{~mm}^{3}$, centered at the Talairach coordinates of the single voxel within left MFG, from which behavioral distraction could be most reliably predicted in experiment 1 [i.e., $(-39,17,19)]$. For each participant, BOLD time course data from each run were then averaged across all voxels within this ROI for additional analysis.

Preparing $f M R I$ data for pretrial signal analysis. ROI data were prepared for pretrial signal analysis in similar manner to experiment 1 , now with the manipulation of display set size taken into account. Nine candlestick predictors were established for each of four conditions: distractor 
present/set size 6, distractor present/set size 10, distractor absent/set size 6 , distractor absent/set size 10. Error trials were modeled as well. Given the nine consecutive candlesticks and a $2 \mathrm{~s}$ TR, an $18 \mathrm{~s}$ duration, posttrial onset was thus modeled. As in experiment 1, predictors for the six motion parameters, white matter signal, and global BOLD signal were included in the model. The regression was then performed, and the residual BOLD time courses were obtained and $z$-transformed for subsequent analysis.

Sorting of trials based on pretrial signal. For each participant, within each run and separately for each level of distractor presence $X$ display set size (i.e., four total conditions), trials were sorted into tertiles of low, medium, and high pretrial signal values, which were sampled from the residual BOLD time courses.

\section{Task-evoked response analysis}

Focusing on the ROI in left MFG, data from experiments 1 and 2 were analyzed to measure the hemodynamic responses evoked by trial stimuli. Preprocessed time course data from each run of each subject were averaged across all voxels of the left MFG ROI, $z$-scored within runs, and then subjected to multiple regression, in which independent candlestick predictors were established for multiple time points of each of the main task conditions. In experiment 1, 11 consecutive time points were modeled for distractor-present and distractor-absent trial types, as well as for error trials. In experiment 2, nine consecutive time points were modeled for the following four conditions: distractor present/set size 6, distractor present/set size 10, distractor absent/set size 6 , and distractor absent/set size 10. Error trials were modeled as well. The regression analyses yielded $\beta$ coefficients for each time point of each trial condition, representing the magnitude of the task-evoked hemodynamic response.

\section{Results}

\section{Experiment 1: whole-brain analysis}

Overall behavior

As described in Materials and Methods, participants performed a feature-based search for a circle among squares in displays in which an irrelevant feature singleton distractor was present or absent (Fig. 1). For each participant, RT data were examined for evidence of behavioral distraction. Error trials were excluded from this analysis, as were trials in which RTs exceeded 3 SD above participants' means for distractor-present and distractor-absent conditions ( 1.0 and $0.9 \%$ of correct trials, respectively). Results showed a significant slowing on distractorpresent trials $(681 \mathrm{~ms})$ compared with distractor-absent trials (665 ms) $\left(t_{(14)}=2.30, p=0.037\right)$, replicating previous studies using this procedure and confirming reliable attention capture (Theeuwes, 1992). Error rates were low and numerically smaller in the distractor-absent condition (3.3\%) than the distractorpresent condition $(4.3 \%)$, although not significantly so $\left(t_{(14)}=\right.$ 1.64, $p=0.12$ ).

\section{Fluctuations in behavioral distraction predicted by pretrial fMRI signal}

To determine whether the degree of distractor interference could be predicted from moment-to-moment fluctuations, pretrial fMRI signal was used, via regression analysis, to predict RT. This was done separately for distractor-present and distractor-absent trials with the following logic: if behavioral distraction varies as a function of pretrial signal, then the relationship between pretrial signal and RT for distractor-present trials must manifest differently than the relationship between pretrial signal and RT for distractor absent trials. For instance, if greater pretrial signal confers smaller behavioral distraction, then RT for distractor-present and distractor-absent trials must converge as pretrial signal increases; likewise, if greater signal confers greater distraction, the RTs must diverge as signal increases. Both possibilities are similar in that they must show a behavioral interaction, and the current

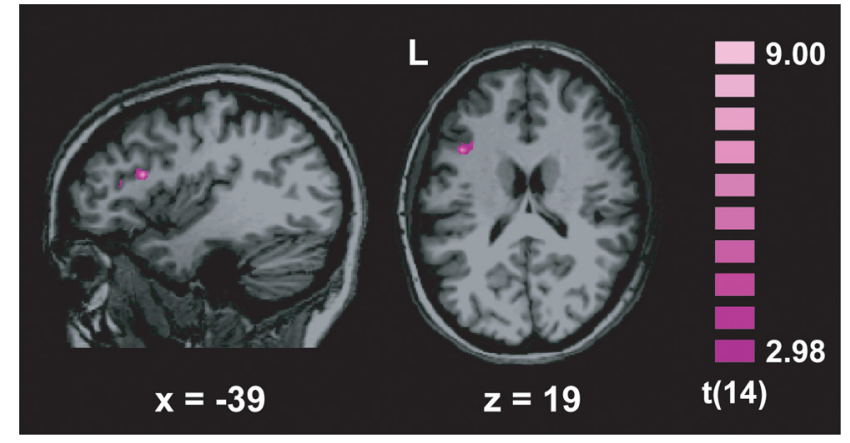

Figure 2. Experiment 1 results. Sagittal and axial slices of a Talairach normalized brain (left and right, respectively), showing a cluster of voxels in left (L) MFG from which pretrial signal reliably predicted fluctuations in behavioral distraction.

regression analysis was designed to identify voxels demonstrating such a pattern, regardless of the directionality.

Results showed that moment-to-moment fluctuations in behavioral distraction could indeed be predicted. One significant cluster of voxels was observed in left MFG, whose statistical peak $\left(t_{(14)}=8.21, p<0.0001\right)$ was located at Talairach coordinates $(-39,17,19)$, with a spatial extent of $298 \mathrm{~mm}^{3}$ (Fig. 2). In this region, RT on distractor-present and distractor-absent trials was shown to converge as pretrial signal increased. That is, trials with greater pretrial signal were associated with reduced distractor interference. These results support the notion that participants are often susceptible to stimulus-driven dominance of attention, but they also undergo periods in which they exert goal-driven control to resist distraction. Note that behavior from this study is not plotted as a function of pretrial activity; this post hoc analysis would not be orthogonal to the whole-brain analysis. Such a plot is provided in experiment 2, in which an ROI-based analysis is applied to an independent set of data.

\section{Experiment 2: independent validation and display set size manipulation}

The goals of this experiment were twofold. First, an ROI-based analysis allowed an independent validation of the experiment 1 results and a more complete characterization of how pretrial signal interacts with attention capture. Second, an important alternative account was considered, as follows. Although the reduction in behavioral distraction at greater pretrial signal in MFG might be assumed to reflect enhanced goal-driven control, such results could have instead been attributable to participants' adoption of a less efficient strategy. Specifically, participants could have opted for a serial search, in which one item (or a few items) is inspected at a time rather than using a parallel search, which simultaneously processes all display locations (Theeuwes, 2004). That is, pretrial signal could reflect the degree to which observers are predisposed to search efficiently (in which efficiency is defined as RT increase per additional display item). Indeed, it is well documented that capture effects are weaker or totally absent during inefficient search (e.g., when RT is slowed by $20 \mathrm{~ms}$ or more per additional display item) (Theeuwes, 1990; Todd and Kramer, 1994; Lamy and Tsal, 1999; Yantis and Egeth, 1999). However, although the adoption of an inefficient search strategy on some trials might bring the observer success in avoiding attention capture, this scenario would be ill described as an improvement in goal-driven control. As a result, the critical question researchers must address is: "can search proceed in parallel and yet, at the same time, be selective toward the task relevant 
Table 1. Experiment 2: pretrial signal mean and SE by tertile, display set size, and distractor condition

\begin{tabular}{|c|c|c|c|c|c|c|c|c|c|c|c|c|}
\hline & \multicolumn{6}{|c|}{ Mean (SD of BOLD signal) } & \multicolumn{6}{|l|}{ SE } \\
\hline & \multicolumn{2}{|l|}{ Low } & \multicolumn{2}{|l|}{ Medium } & \multicolumn{2}{|l|}{ High } & \multicolumn{2}{|l|}{ Low } & \multicolumn{2}{|c|}{ Medium } & \multicolumn{2}{|l|}{ High } \\
\hline & 6 & 10 & 6 & 10 & 6 & 10 & 6 & 10 & 6 & 10 & 6 & 10 \\
\hline Distractor present & -0.757 & -0.783 & -0.040 & -0.047 & 0.675 & 0.700 & 0.036 & 0.038 & 0.034 & 0.033 & 0.037 & 0.037 \\
\hline Distractor absent & -0.765 & -0.770 & -0.055 & -0.048 & 0.713 & 0.714 & 0.038 & 0.039 & 0.033 & 0.031 & 0.039 & 0.042 \\
\hline
\end{tabular}

dimension?" (Theeuwes, 1991, p 184) (see also Leber and Egeth, 2006; Belopolsky et al., 2007). It is thus essential to investigate whether pretrial signal affects search efficiency, a question requiring the manipulation of display set size. To this end, half of the trials contained six display items, whereas the remaining trials contained 10 display items. It might be noted that a pattern of data in which RT increases with larger display set sizes is not a necessary condition for serial search (Townsend, 1971) (but see Woodman and Luck, 1997). However, the expected pattern of data, in which RT does not vary with set size, is generally accepted to confirm parallel search.

To investigate whether and how behavioral distraction and display size effects varied as a function of pretrial activity, pretrial signal values were sampled from the ROI defined in left MFG, and trials were then sorted into tertiles of low, medium, and high pretrial activity. Within each tertile, the sorting procedure yielded pretrial signal means that were comparable across all levels of distractor presence (present and absent) and display set size (6 and 10) (Table 1).

Analysis of RT data excluded error trials as well as trials in which distractor-present and distractor-absent RTs exceeded 3 $\mathrm{SD}$ above each participant's mean in the respective conditions ( 0.7 and $0.8 \%$ of correct trials, respectively). Analysis focused on how effects of distractor presence and display set size changed between the two outermost pretrial signal tertiles (low and high), and a $2 \times 2 \times 2$ repeated measures ANOVA was performed on these conditions. Mean RTs are displayed in Figure 3, including data from the middle tertile, for completeness. Results showed a significant main effect of distractor presence $\left(F_{(1,15)}=13.84, p=\right.$ $0.002)$. This effect interacted significantly with pretrial signal tertile $\left(F_{(1,15)}=6.15, p=0.026\right)$, because distraction was reduced from $36 \mathrm{~ms}$ at the low tertile to $18 \mathrm{~ms}$ at high tertile (when collapsing across display set size). This interaction constitutes an independent validation of the first experiment, showing that trial-by-trial fluctuations in left MFG are predictive of the current degree of attentional control.

Display set size notably did not produce a main effect, nor did it enter into any interactions (all $F$ values $<1.08$ ). RT $\times$ display set size slopes, which were $<3 \mathrm{~ms} /$ item in all conditions, are shown in Table 2 for distractor-present and distractor-absent conditions at all tertiles. Note that slopes appeared slightly greater in the distractor-present conditions; this is attributable to greater distractor interference at larger display set sizes, because the salience of a singleton increases in the presence of more nonsingletons (Bravo and Nakayama, 1990). Thus, slopes in the distractor-absent trials should be emphasized as the unbiased low medium high

Figure 3. Experiment 2 results. RT (top row) and error rates (bottom row) are displayed as a function of distractor presence and 作 the difference between distractor-present and distractor-absent conditions, collapsed across display set size and computed sepa-

measure of search efficiency, and results from these conditions confirm that the reduction of attentional capture at greater pretrial signal was not attributable to changes in search efficiency (i.e., adoption of a serial search). Rather, it appears that greater pretrial signal does indeed reflect increased goal-driven control. No other main effects or interactions were observed (all $F$ values $<1)$.

Error rates, displayed in Figure 3, were analyzed in a similar manner to RT, via a $2 \times 2 \times 2$ ANOVA. These data generally mirrored the RT data (i.e., conditions with faster RT coincided with fewer errors), but no main effects or interactions approached significance.

Although controlling for several aspects of recent trial history, the present analyses did not take into account the role of RT on the previous trial. Because it is possible that previous RT could have influenced the pretrial signal in a way that could account for the present results, additional analysis was performed on experiment 2 data to address this concern. Using linear regression within each run of each participant, variance attributable to previous trial RT was partialed out of the pretrial signal values collected from left MFG (Leber et al., 2008). The sample of residual pretrial signal values was then sorted into tertiles, and consequent behavioral data were analyzed. Results were essentially the same as in the initial analysis, ruling out the possibility that the capacity of MFG to predict attention capture was dependent on previous trial RT (supplemental Fig. 1, available at www.jneurosci.org as supplemental material).

\section{Task-evoked response analysis}

Beyond the capacity of MFG to predict distraction in advance, one might question how this region behaves in response to the task stimuli. In particular, does MFG demonstrate a stimulus- 
Table 2. Experiment 2: RT $\times$ display set size slopes $(\mathrm{ms} /$ item)

\begin{tabular}{|c|c|c|c|}
\hline & \multicolumn{3}{|c|}{ Pretrial signal tertile } \\
\hline & Low & Medium & High \\
\hline Distractor present & 1.5 & 2.1 & 1.5 \\
\hline Distractor absent & -0.8 & 2.9 & -3.4 \\
\hline
\end{tabular}

Experiment 1

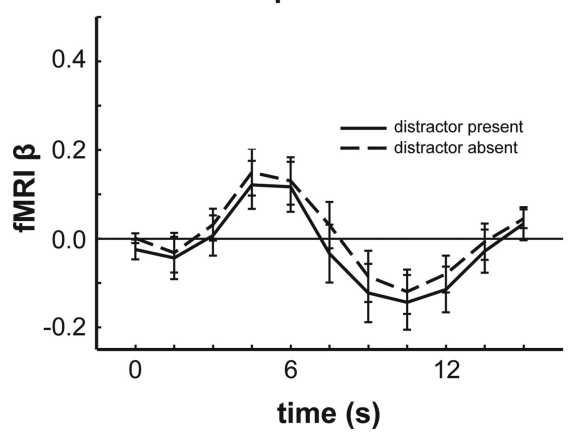

Experiment 2

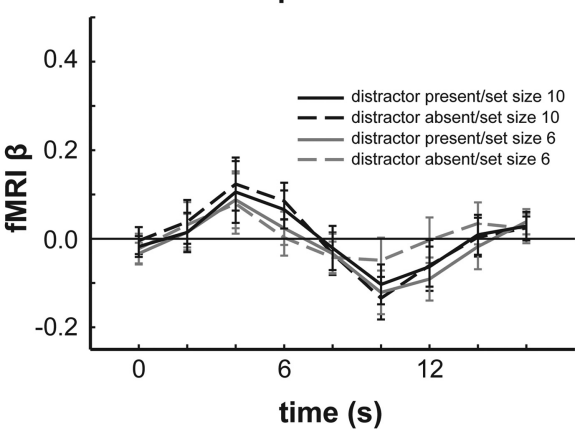

Figure 4. Stimulus-evoked fMRI responses in left MFG. Experiment 1 time courses (TR, $1.5 \mathrm{~s}$ ) are plotted as a function of distractor presence (left). Experiment 2 time courses (TR, $2.0 \mathrm{~s}$ ) are plotted as a function of both distractor presence and display set size (right). Error bars indicate SEM.

evoked response that is sensitive to the presence or absence of a distractor? To address this question, additional ROI-based analysis was performed to examine the hemodynamic response functions in left MFG, for experiments 1 and 2. Results, plotted in Figure 4, were analyzed as follows.

\section{Experiment 1}

Peak hemodynamic responses were compared across distractorpresent and distractor-absent conditions. The fourth and fifth time points, representing the interval of $4.5-7.5 \mathrm{~s}$ after stimulus onset, were selected for inclusion in this analysis. $\beta$ coefficients were averaged across the two time points, separately for distractorpresent and distractor-absent conditions, and were then compared via a group-level $t$ test. Results showed that peak-evoked responses to distractor-present and distractor-absent conditions did not differ significantly $\left(t_{(14)}=0.934, \mathrm{NS}\right)$, suggesting that left MFG is insensitive to the current degree of task-related distraction.

\section{Experiment 2}

The third and fourth time points, representing the interval of $4-8$ $s$ after stimulus onset, were selected for inclusion in this analysis. $\beta$ coefficients were averaged across these two time points separately for the following four conditions: distractor present/set size 6 , distractor present/set size 10 , distractor absent/set size 6 , and distractor absent/set size 10. Resulting coefficients were entered into a 2 (distractor presence) $\times 2$ (set size) ANOVA to determine how the evoked response was influenced by these variables. Results showed no main effect of distractor presence $(F<1)$, replicating the evoked response analysis of experiment 1 and again suggesting that left MFG is insensitive to current task-related distraction. The main effect of set size did reach significance $\left(F_{(1,15)}=7.500, p=0.015\right)$, but the distractor presence $\times$ set size interaction did not $(F<1)$.

\section{Discussion}

Understanding the behavioral properties of attentional control, along with the neural mechanisms subserving them, has long invited intense scrutiny from researchers. However, these efforts have yet to resolve the contentious debate over how stimulusdriven and goal-driven components interact. This study hypoth- esized the existence of within-session intra-individual variability and sought to expose such variability by linking it to fluctuations in neural activity. Such a hypothesis is partly fueled by recent characterizations of variability between individuals in susceptibility to attentional distraction (Fukuda and Vogel, 2009), and it reaches further by probing variability within participants. Results demonstrated that individuals' search behavior is in fact neither all stimulus driven nor all goal driven. Rather, observers routinely shift along a continuum of varying degrees of control, which is predictable in advance via fMRI activity preceding task performance. An alternative account was raised, in which increased pretrial activity does not confer increased goal-driven control but rather is linked to a greater propensity to adopt an inefficient search. Experiment 2 ruled out this account, however, by showing that RT $\times$ display set size functions did not vary with pretrial signal.

It was further observed that the trialevoked responses of the left MFG did not differ between distractor-present and distractor-absent conditions. It thus appears that, although left MFG contributes to fluctuations in the internal state of attentional control, it remains insensitive to the current degree of task-related distraction (for similar dissociations, see Braver et al., 2003; Leber et al., 2008). In interpreting such results, it may be useful to draw an analogy between the left MFG and a sports coach, who provides resources and advance preparation but, unlike the athlete, does not participate in the actual game play.

This study showcases a unique advantage of fMRI in addressing fundamental debates about human behavior. Researchers have studied intra-individual variability for decades, with classical approaches focusing on behavioral measures of RT variability. However, these approaches carry inherent limitations. One such approach, the "stochastic mixture" analysis (Falmagne, 1965; Townsend and Ashby, 1983; Yantis et al., 1991), tests whether participants alternate between two or more discrete mental states, by seeking evidence for distinct bimodal (or multimodal) RT distributions. Demonstrations of stochastic mixtures succeed in verifying alternations between multiple states, but failures to demonstrate mixtures are less interpretable; this is because the approach cannot detect fluctuations that are graded in nature (i.e., waxing and waning on a continuous rather than discrete scale). Indeed, recent behavioral work using a mixture analysis has argued that variation in attentional control, should it exist, is not stochastic in nature (Anderson and Folk, 2010). A second approach, which examines cumulative RT distributions, often by averaging bins of trials into discrete quantiles [e.g., "Vincentizing" (Ratcliff, 1979)] can be sensitive to changes that are graded in nature. This approach has been applied to several studies of attention capture (Theeuwes and Burger, 1998; van Zoest et al., 2004; Ansorge et al., 2005; Gibson and Bryant, 2008; Hickey et al., 2010), but, despite its potential appeal, it carries two central limitations. (1) It assumes that the degree of attentional control must vary as a function of overall RT. That is, if greater goal-driven control does not happen to correspond to faster (or slower) overall responding, then the quantile averaging approach will fail to detect variations, as has been the case in several such analyses. Note that, in the present study, overall RT did not vary significantly as a function of pretrial signal; rather, only the capture 
effect varied. (2) Cumulative RT distribution analysis is susceptible to confounds stemming from trial history that are unrelated to variations in internal control states. That is, the current degree of distractor interference could be attributable to incidental aspects of the stimulus display (e.g., distractors are better ignored when they appear in the same location twice) (Kumada and Humphreys, 2001). As a result, approaches such as quantile averaging are susceptible to systematically placing repetition trials into faster quantiles, which in turn can systematically distort (i.e., shrink) the observed RT interference effect in these quantiles. Consequently, along with the stochastic mixture approach, this approach is arguably not well suited for examination of intraindividual fluctuations in attentional control. The current fMRI technique - prediction from pretrial signal-succeeds because it is uninfluenced by incidental aspects of the stimulus displays that are unrelated to goal-driven control. Moreover, it allows one to predict when resistance to distraction is high or low and also allows examination of the neural substrates mediating such variability. Furthermore, this fMRI technique was able to simultaneously search the whole brain for regions mediating fluctuations in attentional control rather than focusing on one region at a time.

This technique is a natural extension of previous studies of fluctuations in resting state activity, which have contributed much knowledge about intrinsic functional connectivity among brain regions (Biswal et al., 1995; Gusnard and Raichle, 2001; Fox et al., 2005; Vincent et al., 2007). By including task performance, researchers have been able to link presumed baseline fluctuations in neural activity with cognitive processes of interest (Pessoa et al., 2002; Sapir et al., 2005; Turk-Browne et al., 2006; Weissman et al., 2006; Fox et al., 2007; Hahn et al., 2007; Eichele et al., 2008; Leber et al., 2008). For instance, Weissman et al. (2006) reported a network of brain regions whose trial-by-trial activity scaled with overall RT, and they were thus able to characterize the neural signatures of attentional lapses (in which lapses are defined by slow RTs). Leber et al. (2008) further built on the work of Weissman et al. (2006) by isolating a cognitive process more complex than arousal. They identified brain regions that did not correlate with overall RT; rather, these regions selectively predicted the cost of task switching. Similarly, the region of MFG identified presently did not predict overall RT; instead, it selectively predicted the degree of attentional distraction. This pattern of data suggests that one's momentary degree of resistance to feature-based distraction is distinct from his/her current likelihood of attentional lapsing.

Confirming MFG as a key predictor of fluctuations in featurebased attentional control is consistent with several influential models of attention that place a strong emphasis on the role of prefrontal cortex, along with parietal cortex, in establishing goaldriven settings (Miller and Cohen, 2001; Corbetta and Shulman, 2002). These models have drawn considerable support from empirical work (Giesbrecht et al., 2003; Liu et al., 2003; de Fockert et al., 2004; Egner et al., 2008). The majority of these studies measured greater neural activity in subregions of prefrontal cortex in response to instructional cues presented on each trial, encouraging observers to direct attention to a specific aspect of the stimulus (e.g., motion direction or color). Although such cues carry the requirement to modulate the current degree of attentional allocation to features, they also can arguably require cue interpretation and nonspecific task set reconfiguration. These latter cognitive operations have also been well established to recruit prefrontal cortex (Dove et al., 2000; Sohn et al., 2000; Leber et al., 2008), thus potentially complicating inferences about how feature-based control might originate in prefrontal cortex. The current study, however, was unencumbered by task-switching demands, because instructional cues were not used and the search task was the same on each trial (i.e., find the circle). Thus, the current work provides an important confirmation of MFG as a source of feature-based attentional control.

Additional work will be needed to characterize how MFG acts to prioritize visual features in early visual regions. Does MFG serve as a source of enhancement of the target representation, or does it serve as a source of suppression of the salient distractor representation? Much research has discussed the neural consequences of feature-based enhancement and/or suppression in extrastriate visual processing regions (Motter, 1994; Treue and Maunsell, 1996; O'Craven et al., 1997; Chawla et al., 2001; Saenz et al., 2002; Serences and Boynton, 2007), and future efforts to establish direct links between prefrontal sources and posterior consequences of attentional control promise to be fruitful.

\section{References}

Anderson BA, Folk CL (2010) Variations in the magnitude of attentional capture: testing a two-process model. Atten Percept Psychophys 72: 342-352.

Ansorge U, Horstmann G, Carbone E (2005) Top-down contingent capture by color: evidence from RT distribution analyses in a manual choice reaction task. Acta Psychol (Amst) 120:243-266.

Bacon WF, Egeth HE (1994) Overriding stimulus-driven attentional capture. Percept Psychophys 55:485-496.

Belopolsky AV, Zwaan L, Theeuwes J, Kramer AF (2007) The size of an attentional window modulates attentional capture by color singletons. Psychon Bull Rev 14:934-938.

Biswal B, Yetkin FZ, Haughton VM, Hyde JS (1995) Functional connectivity in the motor cortex of resting human brain using echo-planar MRI. Magn Reson Med 34:537-541.

Brainard DH (1997) The psychophysics toolbox. Spat Vis 10:433-436.

Braver TS, Reynolds JR, Donaldson DI (2003) Neural mechanisms of transient and sustained cognitive control during task switching. Neuron 39:713-726.

Chawla D, Rees G, Friston KJ (1999) The physiological basis of attentional modulation in extrastriate visual areas. Nat Neurosci 2:671-676.

Corbetta M, Shulman GL (2002) Control of goal-directed and stimulusdriven attention in the brain. Nat Rev Neurosci 3:201-215.

Dale AM (1999) Optimal experimental design for event-related fMRI. Hum Brain Mapp 8:109-114.

Dale AM, Buckner RL (1997) Selective averaging of rapidly presented individual trials using fMRI. Hum Brain Mapp 5:329-340.

de Fockert J, Rees G, Frith C, Lavie N (2004) Neural correlates of attentional capture in visual search. J Cogn Neurosci 16:751-759.

Dove A, Pollmann S, Schubert T, Wiggins CJ, von Cramon DY (2000) Prefrontal cortex activation in task switching: an event-related fMRI study. Brain Res Cogn Brain Res 9:103-109.

Egner T, Monti JM, Trittschuh EH, Wieneke CA, Hirsch J, Mesulam MM (2008) Neural integration of top-down spatial and feature-based information in visual search. J Neurosci 28:6141-6151.

Eichele T, Debener S, Calhoun VD, Specht K, Engel AK, Hugdahl K, von Cramon DY, Ullsperger M (2008) Prediction of human errors by maladaptive changes in event-related brain networks. Proc Natl Acad Sci U S A 105:6173-6178.

Falmagne JC (1965) Stochastic models for choice reaction time with applications to experimental results. J Math Psychol 2:77-124.

Folk CL, Remington RW, Johnston JC (1992) Involuntary covert orienting is contingent on attentional control settings. J Exp Psychol Hum Percept Perform 18:1030-1044.

Fox MD, Snyder AZ, Vincent JL, Corbetta M, Van Essen DC, Raichle ME (2005) The human brain is intrinsically organized into dynamic, anticorrelated functional networks. Proc Natl Acad Sci U S A 102:9673-9678.

Fox MD, Snyder AZ, Vincent JL, Raichle ME (2007) Intrinsic fluctuations within cortical systems account for intertrial variability in human behavior. Neuron 56:171-184.

Franconeri SL, Simons DJ (2003) Moving and looming stimuli capture attention. Percept Psychophys 65:999-1010. 
Fukuda K, Vogel EK (2009) Human variation in overriding attentional capture. J Neurosci 29:8726-8733.

Gibson BS, Bryant TA (2008) The identity intrusion effect: attentional capture or perceptual load? Vis Cogn 16:182-199.

Giesbrecht B, Woldorff MG, Song AW, Mangun GR (2003) Neural mechanisms of top-down control during spatial and feature attention. Neuroimage 19:496-512.

Goebel R, Esposito F, Formisano E (2006) Analysis of functional image analysis contest (FIAC) data with Brainvoyager QX: from single-subject to cortically aligned group general linear model analysis and selforganizing group independent component analysis. Hum Brain Mapp 27:392-401.

Gusnard DA, Raichle ME (2001) Searching for a baseline: functional imaging and the resting human brain. Nat Rev Neurosci 2:685-694.

Hahn B, Ross TJ, Stein EA (2007) Cingulate activation increases dynamically with response speed under stimulus unpredictability. Cereb Cortex 17:1664-1671.

Hickey C, McDonald JJ, Theeuwes J (2006) Electrophysiological evidence of the capture of visual attention. J Cogn Neurosci 18:604-613.

Hickey C, van Zoest W, Theeuwes J (2010) The time course of exogenous and endogenous control of covert attention. Exp Brain Res 201:789-796.

Kumada T (2001) Feature-based control of attention: evidence for two forms of dimension weighting. Percept Psychophys 63:698-708.

Lamy D, Tsal Y (1999) A salient distractor does not disrupt conjunction search. Psychon Bull Rev 6:93-98.

Lamy D, Carmel T, Egeth HE, Leber AB (2006) Effects of search mode and intertrial priming on singleton search. Percept Psychophys 68:919-932.

Lamy D, Antebi C, Aviani N, Carmel T (2008) Priming of pop-out provides reliable measures of target activation and distractor inhibition in selective attention. Vision Res 48:30-41.

Leber AB, Egeth HE (2006) It's under control: Top-down search strategies can override attentional capture. Psychon Bull Rev 13:132-138.

Leber AB, Turk-Browne NB, Chun MM (2008) Neural predictors of moment-to-moment fluctuations in cognitive flexibility. Proc Natl Acad Sci U S A 105:13592-13597.

Leblanc E, Prime DJ, Jolicoeur P (2008) Tracking the location of visuospatial attention in a contingent capture paradigm. J Cogn Neurosci 20:657-671.

Lien MC, Ruthruff E, Goodin Z, Remington RW (2008) Contingent attentional capture by top-down control settings: converging evidence from event-related potentials. J Exp Psychol Hum Percept Perform 34:509-530.

Liu T, Slotnick SD, Serences JT, Yantis S (2003) Cortical mechanisms of feature-based attentional control. Cereb Cortex 13:1334-1343.

Miller EK, Cohen JD (2001) An integrative theory of prefrontal cortex function. Annu Rev Neurosci 24:167-202.

Motter BC (1994) Neural correlates of attentive selection for color or luminance in extrastriate area V4. J Neurosci 14:2178-2189.

Müller HJ, Geyer T, Zehetleitner M, Krummenacher J (2009) Attentional capture by salient color singleton distractors is modulated by top-down dimensional set. J Exp Psychol Hum Percept Perform 35:1-16.

O'Craven KM, Rosen BR, Kwong KK, Treisman A, Savoy RL (1997) Voluntary attention modulates fMRI activity in human MT-MST. Neuron 18:591-598.

Olivers CN, Humphreys GW (2003) Attentional guidance by salient feature singletons depends on intertrial contingencies. J Exp Psychol Hum Percept Perform 29:650-657.

Pelli DG (1997) The VideoToolbox software for visual psychophysics: transforming numbers into movies. Spat Vis 10:437-442.

Pessoa L, Gutierrez E, Bandettini P, Ungerleider L (2002) Neural correlates of visual working memory fMRI amplitude predicts task performance. Neuron 35:975-987.

Pinto Y, Olivers CNL, Theeuwes J (2005) Target uncertainty does not lead to more distraction by singletons: intertrial priming does. Percept Psychophys 67:1354-1361.

Ratcliff R (1979) Group reaction time distributions and an analysis of distribution statistics. Psychol Bull 86:446-461.

Saenz M, Buracas GT, Boynton GM (2002) Global effects of feature-based attention in human visual cortex. Nat Neurosci 5:631-632.

Sapir A, d'Avossa G, McAvoy M, Shulman GL, Corbetta M (2005) Brain signals for spatial attention predict performance in a motion discrimination task. Proc Natl Acad Sci U S A 102:17810-17815.

Serences JT, Boynton GM (2007) Feature-based attentional modulations in the absence of direct visual stimulation. Neuron 55:301-312.

Serences JT, Shomstein S, Leber AB, Golay X, Egeth HE, Yantis S (2005) Coordination of voluntary and stimulus-driven attentional control in human cortex. Psychol Sci 16:114-122.

Sohn MH, Ursu S, Anderson JR, Stenger VA, Carter CS (2000) The role of prefrontal cortex and posterior parietal cortex in task switching. Proc Natl Acad Sci U S A 97:13448-13453.

Talairach J, Tournoux P (1988) Co-planar stereotaxic atlas of the human brain: 3-dimensional proportional system: an approach to cerebral imaging. New York: Thieme.

Theeuwes J (1990) Perceptual selectivity is task dependent: evidence from selective search. Acta Psychol (Amst) 74:81-99.

Theeuwes J (1991) Cross-dimensional perceptual selectivity. Percept Psychophys 50:184-193.

Theeuwes J (1992) Perceptual selectivity for color and form. Percept Psychophys 51:599-606.

Theeuwes J (2004) Top-down search strategies cannot override attentional capture. Psychon Bull Rev 11:65-70.

Theeuwes J, Burger R (1998) Attentional control during visual search: The effect of irrelevant singletons. J Exp Psychol Hum Percept Perform 24:1342-1353.

Todd S, Kramer AF (1994) Attentional misguidance in visual search. Percept Psychophys 56:198-210.

Townsend JT, Ashby FG (1983) The stochastic modeling of elementary psychological processes. Cambridge, UK: Cambridge UP.

Treue S, Maunsell JH (1996) Attentional modulation of visual motion processing in cortical areas MT and MST. Nature 382:539-541.

Turk-Browne NB, Yi DJ, Chun MM (2006) Linking implicit and explicit memory: common encoding factors and shared representations. Neuron 49:917-927.

van Zoest W, Donk M, Theeuwes J (2004) The role of stimulus-driven and goal-driven control in saccadic visual selection. J Exp Psychol 30:746-759.

Vincent JL, Snyder AZ, Fox MD, Shannon BJ, Andrews JR, Raichle ME, Buckner RL (2006) Coherent spontaneous activity identifies a hippocampal-parietal memory network. J Neurophysiol 96:3517-3531.

Vincent JL, Patel GH, Fox MD, Snyder AZ, Baker JT, Van Essen DC, Zempel JM, Snyder LH, Corbetta M, Raichle ME (2007) Intrinsic functional architecture in the anaesthetized monkey brain. Nature 447:83-86.

Weissman DH, Roberts KC, Visscher KM, Woldorff MG (2006) The neural bases of momentary lapses in attention. Nat Neurosci 9:971-978.

Woodman GF, Luck SJ (1999) Electrophysiological measurement of rapid shifts of attention during visual search. Nature 400:867-869.

Yantis S, Egeth HE (1999) On the distinction between visual salience and stimulus-driven attentional capture. J Exp Psychol Hum Percept Perform 25:661-676.

Yantis S, Jonides J (1984) Abrupt visual onsets and selective attention: evidence from visual search. J Exp Psychol Hum Percept Perform 10: 601-621.

Yantis S, Meyer DE, Smith JE (1991) Analyses of multinomial mixture distributions: new tests for stochastic models of cognition and action. Psychol Bull 110:350-374. 